\title{
Kapitel 4 - Der Grundreiz der Untersuchung
}

Im Unterschied zu solchen Verfahren der Gruppendiskussion, in denen der Grundreiz lediglich der Initialisierung einer Diskussion dient und für die Analyse kaum von Bedeutung ist, ist es bei der Methode der fokussierten Gruppendiskussion notwendig, den Stimulus genauer zu analysieren. Dies liegt daran, dass er das zentrale Referenzereignis darstellt, auf das sich in den Diskussionen und den darauffolgenden Interviews explizit und implizit immer wieder bezogen wird. Dies soll hier in zwei Schritten geschehen. $\mathrm{Zu}$ nächst wird das Gedicht »Was gesagt werden muss« analysiert (Kapitel 4.1), sodann der Bericht des ZDF (Kapitel 4.2). Der Fokus der Analyse liegt hierbei auf den gesprochenen Worten, jedoch werden auch einige visuelle Elemente des Medienclips beschrieben.

Um die inhaltliche Analyse in geordneter Weise durchzuführen, wird in Anschluss an diskurstheoretische Überlegungen von Jürgen Habermas heuristisch zwischen konstativen, evaluativen und expressiven Äußerungen unterschieden. Unter konstativen Sprechakten werden solche Äußerungen verstanden, mit denen sich auf die objektive Welt überprüfbarer Sachverhalte bezogen wird. Mit solchen Sprechakten ist nach Habermas der implizite Anspruch verbunden, sachlich angemessene und intersubjektiv nachvollziehbare Aussagen über eine gesellschaftliche oder politische Realität zu treffen. Unter evaluativen Sprechakten werden demgegenüber Äußerungen verstanden, mit denen Bewertungen vorgenommen werden, die auf kollektiv geltenden Normen rekurrieren. Drittens wird sich in expressiven Äußerungen auf die subjektive Welt des persönlichen Erlebens bezogen und also auch etwas über sich selbst gesagt (vgl. Habermas 1971: 111f.). 


\subsection{Analyse des Gedichtes "Was gesagt werden muss"}

Zunächst wird auf die besondere Form des Textes und dann auf die Inhalte eingegangen. Hieran schließt eine kritische Evaluation des Gedichtes an. Bevor dies aber geschieht, wird es hier zunächst im vollen Wortlaut wiedergegeben (vgl. Grass 2012):

\section{Was gesagt werden muss}

Warum schweige ich, verschweige zu lange, was offensichtlich ist und in Planspielen geübt wurde, an deren Ende als Überlebende wir allenfalls Fußnoten sind.

Es ist das behauptete Recht auf den Erstschlag, der das von einem Maulhelden unterjochte und zum organisierten Jubel gelenkte iranische Volk auslöschen könnte,

weil in dessen Machtbereich der Bau einer Atombombe vermutet wird.

Doch warum untersage ich mir, jenes andere Land beim Namen zu nennen, in dem seit Jahren - wenn auch geheimgehalten ein wachsend nukleares Potential verfügbar aber außer Kontrolle, weil keiner Prüfung zugänglich ist?

Das allgemeine Verschweigen dieses Tatbestandes, dem sich mein Schweigen untergeordnet hat, empfinde ich als belastende Lüge und Zwang, der Strafe in Aussicht stellt, sobald er mißachtet wird; das Verdikt »Antisemitismus« ist geläufig.

Jetzt aber, weil aus meinem Land, das von ureigenen Verbrechen, die ohne Vergleich sind, Mal um Mal eingeholt und zur Rede gestellt wird, wiederum und rein geschäftsmäßig, wenn auch 
mit flinker Lippe als Wiedergutmachung deklariert, ein weiteres U-Boot nach Israel geliefert werden soll, dessen Spezialität darin besteht, allesvernichtende Sprengköpfe dorthin lenken zu können, wo die Existenz einer einzigen Atombombe unbewiesen ist, doch als Befürchtung von Beweiskraft sein will, sage ich, was gesagt werden muß.

Warum aber schwieg ich bislang?

Weil ich meinte, meine Herkunft, die von nie zu tilgendem Makel behaftet ist, verbiete, diese Tatsache als ausgesprochene Wahrheit dem Land Israel, dem ich verbunden bin und bleiben will, zuzumuten.

Warum sage ich jetzt erst, gealtert und mit letzter Tinte:

Die Atommacht Israel gefährdet den ohnehin brüchigen Weltfrieden?

Weil gesagt werden muß,

was schon morgen zu spät sein könnte; auch weil wir - als Deutsche belastet genug Zulieferer eines Verbrechens werden könnten, das voraussehbar ist, weshalb unsere Mitschuld durch keine der üblichen Ausreden zu tilgen wäre.

Und zugegeben: ich schweige nicht mehr, weil ich der Heuchelei des Westens überdrüssig bin; zudem ist zu hoffen, es mögen sich viele vom Schweigen befreien, den Verursacher der erkennbaren Gefahr zum Verzicht auf Gewalt auffordern und gleichfalls darauf bestehen, daß eine unbehinderte und permanente Kontrolle des israelischen atomaren Potentials und der iranischen Atomanlagen durch eine internationale Instanz von den Regierungen beider Länder zugelassen wird. 


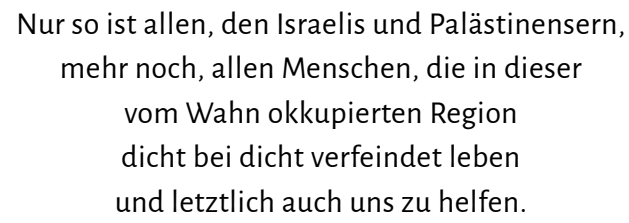

\subsubsection{Die besondere Form des Gedichts}

Viele Kommentator*innen haben dem Gedicht seinen lyrischen Charakter abgesprochen und »Was gesagt werden muss« als politische Verlautbarung bezeichnet. Diese Interpretationen sind insofern nachvollziehbar, als sich das Gedicht im Wesentlichen nicht durch das auszeichnet, was der Linguist Roman Jakobson als »Literarizität « bezeichnet, die auf die "poetischen Funktion« bzw. die »Ausrichtung auf die Sprache selbst« (vgl. Nünning 2013: 453) zurückzuführen ist. ${ }^{1}$ Zwar gibt es bei Grass Elemente der poetischen Funktion, z.B. Wiederholungen und andere dramaturgische Elemente, allerdings überwiegen andere Sprachfunktionen, insbesondere die konative Funktion als Appell an die Adressat*innen. Auf der anderen Seite kann argumentiert werden, dass es sich bei dem Text um ein politisches Gedicht und damit um eine Sonderform der Lyrik handelt, die darauf abzielt, Einsichten in die politische Wirklichkeit zu fördern (vgl. Hinderer 2012: o.S.). Dies geht laut Dieter Lamping bei »Was gesagt werden muss« zudem mit der Beanspruchung einer säkularisierten Form des »antik-religiösen Sehertums « einher (Lamping 2012: o.S.). Grass behauptet hierbei, Einsichten in die politische Realität und ihre zukünftige Entwicklung gewonnen zu haben, die nun anderen kundgetan werden sollen. Aber diesbezüglich ist festzuhalten, dass - auch wenn »Was gesagt werden muss « als poetisches Werk betrachtet wird - es als Grenzfall politischer Lyrik bezeichnet werden sollte. Dies hat vor allem mit dem hybriden Charakter des Textes zu tun. Körte zufolge bestehe eine Spannung zwischen der »kodifizierten, hermetischen Form des Sprechens« und dem Anschluss an die »Schreibgepflogenheiten von Leitartikeln« (Körte 2014: 2). Und

1 Mein Dank gilt Annemarie Tastel für die literaturwissenschaftliche Erläuterung dieser zentralen Eigenschaft des Gedichts. 
Heinrich Detering betont, dass damit der implizite Anspruch einhergehe, gesellschaftliche Realitäten nicht künstlerisch, sondern objektiv zu beschreiben (vgl. Detering 2012: o.S.). Dementsprechend ist es angemessen, zumindest die im Gedicht aufgestellten Behauptungen, wie andere Formen der politischen Rede auch, zu untersuchen und zu kritisieren.

\subsubsection{Konstative, evaluative und expressive Äußerungen}

Zunächst sollen hier die wichtigsten im Text aufgestellten Behauptungen (d.h. konstative Äußerungen), mit denen beansprucht wird eine politische Realität angemessen abzubilden, zusammengefasst werden. Hierzu gehören insbesondere folgende:

a) Deutschland beabsichtige ein U-Boot an Israel zu liefern, das als Massenvernichtungswaffe einsetzbar sei.

b) Israels »atomares Potential« sei keiner internationalen Kontrolle zugänglich.

c) Israel behaupte gegenüber dem Iran ein »Recht auf den Erstschlag", was bei dessen Durchführung die "Auslöschung« des iranischen Volkes sowie der Zerstörung des »ohnehin brüchigen Weltfriedens« bedeute. Die Wörter »Erstschlag«, »Planspiel«, »Auslöschung« und »wir Überlebende als Fußnoten « verweisen darauf, dass dieser militärische Angriff als atomarer verstanden wird, und es wird nahegelegt, dass dieser in seinem Effekt globale und totale Ausmaße annehmen könne.

d) Es sei eine bloße Vermutung, dass das iranische Regime an der Entwicklung von Atomwaffen arbeite. Das verbale Verhalten des damaligen iranischen Präsidenten Mahmud Ahmadinedschads sei als Ausdruck von "Maulheldentum«, d.h. als Fassade zu verstehen, hinter der sich keine tatsächlichen Absichten verbürgen.

e) Es werde verschwiegen, dass Israel eine Bedrohung darstelle und wahrhaftige Formen des Sprechens über Israel - also >Israelkritik - setze sich üblicherweise der Gefahr aus, als antisemitisch verurteilt zu werden (»das Verdikt >Antisemitismus ist geläufig().

Neben diesen Behauptungen finden sich im Gedicht mehrere moralische Bewertungen (d.h. evaluative Äußerungen), die mit politischen Forderungen verbunden werden: 
a) Es handle sich bei der wahrscheinlichen Vernichtung des iranischen Volks um einen kriminellen Akt (ein »Verbrechen«).

b) Die für diesen kriminellen Akt verwendeten Waffensysteme stammten aus Deutschland, weshalb Deutsche als »Zulieferer« eine »Mitschuld«träfe.

c) Über die von Israel ausgehenden Gefahr müsse überhaupt erst ein öffentlicher Diskurs etabliert werden, da diese Tatsache beschwiegen würde (»es mögen sich viele vom Schweigen befreien«).

d) Das »israelische atomare Potential«, sowie iranische Atomanlagen, sollten unter internationale Kontrolle gestellt werden.

Diese von Grass artikulierten Forderungen machen deutlich, dass dem Gedicht ein ausgesprochen starker Appellcharakter ${ }^{2}$ (vgl. von Thun 1981: 12ff.) zukommt, der bereits im Titel deutlich wird. Mit der Forderung, dass etwas gesagt werden muss, wird eine dringliche, imperativische Forderung aufgestellt. Diese Dringlichkeit der Thematisierung einer von Israel ausgehenden Gefahr wird hierbei in der sechsten Strophe mit der Behauptung eines Schuldzusammenhangs verbunden, der sich im Falle eines nicht-Handelns für die deutschen Adressaten als »Zulieferer« ergeben würde. Das mögliche Handeln oder Nichthandeln >der Deutschen« wird hierbei mit der Wendung »als Deutsche belastet genug« in direktem Zusammenhang zur mit Schuld belasteten Wir-Gruppe, aufgrund des Nationalsozialismus, gestellt. Der moralische Appell besteht also auch darin, dass die Deutschen Konsequenzen aus der nationalsozialistischen Vergangenheit ziehen und sich heute moralisch verhalten sollen. Der Appellcharakter des Gedichts wird dann nochmals in der vorletzten Strophe deutlich, in der dazu aufgefordert wird, »es mögen sich viele vom Schweigen befreien«. Auch hierbei handelt es sich um Vokabular, das aus Erinnerungsdiskursen bekannt ist. Die implizite Forderung, die mit dem Gedicht verbunden ist, ist, dass Israel nicht trotz, sondern wegen der nationalsozialistischen Vergangenheit politisch Einhalt zu gebieten sei.

Drittens können im Gedicht verschiedene expressive Äußerungen identifiziert werden, mit denen Grass etwas über sich selbst sagt. Hierzu gehören insbesondere die wiederholten Formulierungen »verschweige«, »untersage mir [...] zu nennen« und »schweige nicht mehr«. Das Lyrische Ich spricht hier vom eigenen (bisherigen) Verschweigen, um dieses im gleichen Zuge zu aussage und des Beziehungsaspekts eine der vier Seiten einer Nachricht. 
brechen. Grass stellt sich dadurch als Sprecher dar, der bisher nicht wahrhaftig gehandelt habe, dies aber nun tue. Diese Behauptung einer neuen Wahrhaftigkeit wird insbesondere dadurch konturiert, dass die Rechtfertigungsversuche des »Westens«, sowie das vermeintlich hegemoniale Schweigen über die von Israel ausgehende Gefahr, als unwahrhaftiges Handeln (»Heuchelei«) dargestellt wird. Hierbei wird insbesondere die Unwahrhaftigkeit des Schweigens problematisiert und dieses damit gewissermaßen zur Abwehrhandlung deklariert (siehe Kapitel 2.2). Grass bietet hierbei zwei Erklärungen für das eigene und das kollektive Schweigen an. Zum einen habe er aus der Annahme heraus geschwiegen, dass es ein mit seiner deutschen Herkunft verbundener »Makel« es »verbiete«, Israel die »Wahrheit« zuzumuten. Zum anderen aber, sei das Schweigen erzwungen, da wahrhaftiges Sprechen als vermeintlicher »Antisemitismus « verurteilt würde. Ein dramaturgischer Höhepunkt des Gedichts liegt hierbei in Strophe 7, wo das Lyrische Ich "mit letzter Tinte« die Quintessenz dessen zum Ausdruck bringt, was es wahrhaftig sagen will und muss, nämlich, dass die »Atommacht Israel« den »ohnehin brüchigen Weltfrieden« bedrohe.

\subsubsection{Evaluation des Gedichts}

Die Evaluation des Gedichts erfolgt nun in drei Schritten. Zunächst geht es darum, die konstativen Behauptungen von Grass auf ihren Realitätsgehalt hin zu befragen. Danach soll das Gedicht aus antisemitismuskritischer Perspektive betrachtet werden.

\section{Überprüfung der konstativen Behauptungen}

Außer Frage steht zunächst, dass Deutschland im März 2012 tatsächlich die Lieferung eines U-Bootes der Dolphin-Klasse an das israelische Militär angekündigt hatte und dass die Wahrscheinlichkeit, dass dieses U-Boot mit Atomwaffen ausgerüstet wird als wahrscheinlich gelten konnte. Demgegenüber wird bei der Berücksichtigung fachwissenschaftlicher Debatten deutlich, dass die anderen im Gedicht vorgenommenen konstativen Behauptungen als irreführend, faktisch falsch oder realitätsverzerrend zu bezeichnen sind. ${ }^{3} \mathrm{Zu}$ -

Ein systematischer Überblick über die mit Grass Behauptungen korrespondierende Faktenlage, wie sie insbesondere in der Forschung zu den internationalen Beziehungen und der Rüstungsforschung diskutiert wird, ist bei Gert Krell und Harald Müller (dies. 2012) zu finden. 
nächst ist darauf hinzuweisen, dass es zwar stimmt, dass israelische Atomwaffen von keiner internationalen Organisation kontrolliert werden, dass dies aber auch auf keinen anderen Staat der Welt zutrifft, auch nicht in Hinsicht auf konventionelle Waffen. Die Suggestion, dass durch entsprechende Kontrollen ein geltender Standard durchgesetzt werden könnte, ist deshalb irreführend (vgl. Jahn 2012: 8). Weiterhin ist es falsch, dass Israel jemals das Recht auf einen atomaren Angriff (»Erstschlag«) behauptet habe. Es kann hierbei als ausgeschlossen gelten, dass Israel den Iran mit atomaren Waffen, die es seit etwa 1967 besitzt, angegriffen hätte. Denn die israelischen Atomwaffen sind, wie die Unterseeboote auch, nicht als Erstschlags-, sondern Vergeltungswaffen konzipiert, weshalb ihre Existenz auch nicht offiziell bestätigt wird (vgl. Müller 2012: 33f.). Stattdessen wäre ein israelischer Angriff auf iranische Anlagen zur Atomanreicherung - der dem israelischen Expräsidenten Shimon Peres von der Netanjahu-Regierung tatsächlich ernsthaft in Erwägung gezogen worden war (vgl. Bertrand 2016) - mit konventionellen Waffen geführt worden. Dass die iranische Bevölkerung hierbei unmittelbar als Opfer betroffen gewesen wäre, kann dabei allerdings als sehr unwahrscheinlich gelten. Weiterhin lässt sich festhalten, dass ein entsprechender Konflikt zwar mit der reellen Gefahr eines Regionalkriegs, aber nicht mit der Gefahr eines Weltkrieges verbunden gewesen wäre (vgl. Krell 2012: 5ff., 17, vgl. auch Jahn 2012: 11).

Bezüglich der Grass'schen Aussagen über das iranische Atomprogramm lässt sich festhalten, dass bereits zum Zeitpunkt der Veröffentlichung des Gedichts viele Indizien darauf hinwiesen, dass der Iran seit den 2000er Jahren tatsächlich an einer Atomwaffe gearbeitet hat und es sich hierbei also zumindest nicht um aus der Luft gegriffene Behauptungen handelt, wie die Wendung »als Befürchtung von Beweiskraft« nahelegt (vgl. IAEA 2011). ${ }^{4}$ Wenngleich aber auch das israelische Militär im Jahr 2012 davon ausging, dass das iranische Regime nicht die notwendigen Schritte unternahm, um entsprechende Waffen zeitnah zu produzieren (vgl. Bertrand 2012), so ist die Behauptung, dass es sich beim damaligen Präsidenten Mahmud Ahmadinedschad Anzeichen dafür vor, dass der Iran mindestens bis ins Jahr 2010 an einem nuklearen Sprengkörper gearbeitet hat. Der Bericht spricht von verschiedenen, für den Bau von Atomwaffen relevanten, Computermodellen, Experimenten mit Zündvorrichtungen und der Beschaffung verschiedener Bauteile, die für den Bau einer solchen Waffe notwendig sind (vgl. ders.). 
um einen »Maulhelden « handle, als grobe Verharmlosung der Bedrohungslage Israels zu verstehen. Als Maulheld kann eine Person gelten, die gerne mit ihren Taten prahlt, tatsächlich aber kaum etwas entsprechendes tut. Demgegenüber ist festzuhalten, dass die im Iran dominierenden religiösen und politischen Eliten, allen voran Ajatollah Khamenei, die politische Führung auf die Vernichtung des israelischen Staats verpflichtet. Der "Zionismus« wird von diesen Akteuren nicht als gewöhnlicher Gegner betrachtet, »sondern als Grundübel, das für nahezu alle Probleme in der Welt verantwortlich sei und dessen Auslöschung den Weg zur Erlösung bereite« (Grigat 2018: 2014). Die dämonisierende und antisemitische Darstellung Israels als "Krebsgeschwür« sowie die Forderung nach dessen Zerstörung ist hierbei als traditioneller Bestandteil iranischer innen- und außenpolitischer Rhetorik seit der islamischen Revolution zu betrachten. Stephan Grigat zufolge stellt sie neben »klassischer Judenfeindschaft, einer verschwörungstheoretischen und projektiven Weltsicht « sowie »Holocaustleugnung und -relativierung (ebd.: 200) eine der bestimmenden Formen des Antisemitismus iranischer Islamisten dar. Praktischen Niederschlag fand und findet die antisemitische und antizionistische Agitation insbesondere in der materiellen Unterstützung islamistischer Milizen, wie dem islamischen Dschihad, der Hisbollah und auch der Hamas und damit von politischen Terrororganisationen, deren Gewalt sehr konkrete Folgen für die israelische Bevölkerung hat (vgl. Krell 2012: 7, 10). Mit der Fokussierung auf eine einzelne diktatorische Figur, die nicht ernst zu nehmen sei, geht also im Gedicht eine Nicht-Thematisierung der politischen Verhältnisse einher, dessen Teil sie ist. Die argumentative Figur, den Antisemitismus von der iranischen Politik zu lösen, um diesen auf eine einzelne führende Person zu projizieren, wo er wiederum als nicht ernstzunehmendes Gerede erscheint, lässt sich als Form der externalisierenden Abspaltung und zugleich als Leugnung von Antisemitismus beschreiben.

Zuletzt ist darauf hinzuweisen, dass auch die Behauptung, dass über die Existenz israelischer Atomwaffen nicht offen gesprochen werden könne, bzw. eine Kritik an den mit einem Präventivangriff verbundenen Gefahren als antisemitisch gebrandmarkt werde, faktisch falsch ist. So ist die Existenz israelischer Atomwaffen ein Gegenstand, über den beispielsweise in der Rüstungsforschung seit Jahren rege diskutiert wird. Auch wurde über einen möglichen Angriff Israels auf den Iran bereits zum Zeitpunkt der Veröffentli- 
chung von »Was gesagt werden muss « eine politische Debatte geführt. ${ }^{5}$ Hierbei hatte im Februar 2012 der damalige deutsche Verteidigungsminister Thomas de Maizière auf der Münchener Sicherheitskonferenz Israel vor möglichen "Abenteuern« gewarnt (vgl. dpa/AZ 2012, o.S.), und wurde hierfür weder kritisiert noch als antisemitisch bezeichnet (vgl. Riebe 2012, o.S.). Dementsprechend kann von einer gesellschaftlichen Tabuisierung dieses Themas keine Rede sein.

\section{Antisemitischer Charakter des Gedichts}

Im Fall des Gedichts »Was gesagt werden muss « gibt es verschiedene Eigenschaften, die es als antisemitischen Text auszeichnen. Diesbezüglich ist zunächst festzuhalten, dass in dem Gedicht, wie für den Antisemitismus typisch, eine dichotome Konstruktion von Täter- und Opfergruppen vorgenommen wird, die von internen Widersprüchen bereinigt ist (vgl. Holz 2007: 39). Israel bzw. Israelis werden hierbei als (potenziell) Schuldige, die Opfergruppe, das iranische Volk, als deren Opfer präsentiert. Dementsprechend wird auch nicht zwischen israelischer Regierung und Volk unterschieden, und stattdessen ganz allgemein von dem »Land«, »Israel« oder den »Israelis« gesprochen (vgl. Grass 2012). Auf iranischer Seite wird hingegen zwischen Volk und Führung differenziert. Ich folge hierbei Müller, der die von Grass im Nachhinein formulierte Relativierung, er habe das Gedicht lediglich auf die israelische Regierung (bzw. Netanjahu) beziehen wollen, als »nachträgliche Schutzbehauptung« (Müller 2012:33) und damit als Ausdruck von Abwehrverhalten versteht. Die Darstellung Ahmadinedschads als »Maulheld« ohne Mittel, Möglichkeiten oder tatsächlichem Willen Israel zu schaden, führt hierbei zu einer Absicherung des Bildes von Israel als Täterland. Denn würde eine vom iranischen Regime ausgehende Bedrohung anerkannt, wäre Israel nicht mehr eindeutig als alleiniger Ausgangspunkt der bestehenden Gefahrenlage identifizierbar. Mit dieser "moralischen, anklagenden Formierung der Dichotomie« (ebd.), die für antisemitische Texte typisch sind, geht zudem eine besondere Form

So berichteten beispielsweise die Presse im Zusammenhang von Berichten über die angestrebte Lieferung des U-Bootes und der Drohungen Israels gegen den Iran, dass sich die Bundesregierung auf einen solchen Angriff bereits einstelle. In der Berichterstattung über diese Ereignisse wurde in der Tagespresse zudem darüber spekuliert, dass das Boot mit Atomwaffen ausstattbar sei (vgl. dpa/sara 2012, n-tv.de 2012, Berlin afp 2012, Birnbaum/Sirleschtov 2012). Der Spiegel hatte bereits im Jahr 2003 darüber berichtet, Israel plane die U-Boote mit Unterwasserrampen für Atomraketen auszustatten (vgl. Spiegel Online 2003b). 
der personalisierenden Erklärung einher (vgl. ebd.: 39f.). Grass verweist nicht einfach auf die tatsächlich mögliche Gefahr eines Eskalationsprozesses des israelisch-iranischen Konfliktes, stattdessen findet in >den Israelis bzw. >Israel eine Personifikation der möglichen Gefahren statt und Israel bzw. Israelis werden letztendliche als potenzielle Verbrecher dargestellt.

Bereits in der Einleitung wurde festgestellt, dass es im Gedicht zu einer Übertragung antisemitischer Stereotype auf den israelischen Staat kommt. Im Fall von »Was gesagt werden muss" wird Israel bzw. Israelis zwar nicht direkt abgesprochen eine Nation wie andere Nationen zu sein (vgl. ebd.: 46), und Israel wird auch nicht im Sinne einer systematischen Verschwörungstheorie zum Übel der Welt erklärt. Allerdings ist das Gedicht von apokalyptischen Tönen geprägt. Israel bzw. Israelis werden hierbei letztlich, wenn man es gewähren ließe, als zerstörerisch und gemeingefährlich, als kriminell bzw. als Akteur dargestellt, der die Massenvernichtung von Menschen mindestens billigend in Kauf nimmt, um seine politischen Ziele zu verfolgen. Zugleich wird auch auf indirekte Weise behauptet, dass Israel eine Gefahr für die Deutschen sein könnte. So spricht Grass von vermeintlich durchgeführten »Planspiele[n], [...] an deren Ende als Überlebende wir allenfalls Fußnoten sind« (Grass 2012). Israel erscheint damit, wie Jüd"innen im traditionellen Antisemitismus, als Gefahr für die gesamte Menschheit.

Dagegen ließe sich einwenden, dass im traditionellen Antisemitismus >die Juden « üblicherweise nicht als Gruppe imaginiert wird, die ihre Macht unmittelbar ausübt, sondern auf vermittelte Weise, d.h. durch Intrigen, Strippenzieherei, politische Manipulation usw. Wäre es deshalb nicht eventuell angemessener, Grass dämonisierende Äußerungen über Israel als Ausdruck von "Israelfeindlichkeit« (vgl. Globisch 2008: 5587ff.) zu verstehen? Dieser Einwand ist bedenkenswert, allerdings bedient Grass noch eine andere Form der Argumentation, die insbesondere für einen schuldabwehrenden Antisemitismus typisch ist. In Kapitel 2.4 wurde diesbezüglich gezeigt, dass für den sekundären Antisemitismus das Motiv der Aufrechnung von großer Bedeutung ist und sich z.B. in Form von NS-Vergleichen dokumentiert, die auch auf den jüdischen Staat bezogen werden können (vgl. Heyder et al. 2005: 149; Kloke 2005: o.S.). Dies findet im Gedicht auf indirekte Weise statt. Die deutsche Wir-Gruppe als »Überlebende« und »Fußnoten der Geschichte« (also als Vergessene) wie auch das iranische Volk als »ausgelöschtes" werden hierbei durch Worte beschrieben, die dem sprachlichen Register des Holocaustdis- 
kurses entstammen. ${ }^{6}$ Durch die Identität dieser sprachlichen Ausdrücke wird hierbei die mögliche Vernichtung des iranischen Volks in unmittelbare Nähe des Genozids an den Jüd*innen gerückt. Doch im Grunde geht das Gedicht über eine Gleichsetzung von NS-Tätern und Israel weit hinaus, wenn berücksichtigt wird, dass Israel nicht weniger als die potenzielle Auslöschung von dutzenden Millionen Menschen angelastet wird.

Abschließend sei darauf hinzuweisen, dass Grass seine Argumentation als Tabubrecher in die öffentliche Debatte einbringt, und damit ein typisches antisemitisches Verhaltensmuster reproduziert (vgl. Kapitel 2.1). Bereits der Titel lässt sich hierbei, der Literaturwissenschaftlerin Monika Körte zufolge, als "vereindeutigende Arbeit« des Satzes »Man muss doch noch sagen dürfen...« verstehen (vgl. Mona Körte 2012: 2). Während dieser Satz den Tabubruch durch den »konjunktivischen Modus« allerdings nur berührt »so wählt Grass einen Aussagesatz, eigentlich einen Imperativ, der das Tabu überschreitet«(ebd.). Im Gedicht selbst wird die Notwendigkeit dieses Tabubruchs dann durch die Behauptung plausibilisiert, dass falsche Antisemitismusvorwürfe »geläufig« (also normalisiert) seien. Diese Figur lässt sich auch als Ausdruck einer Kritik antizipierenden Abwehrstrategie interpretieren, insofern sie durch die Vorwegnahme und Herabsetzung möglicher Antisemitismuskritik darauf abzuzielen scheint, die Chance zu erhöhen, sich im Falle von Kritik als Opfer ungerechter Vorwürfe darstellen zu können und dadurch Unterstützer*innen zu mobilisieren (vgl. Süselbeck 2012: o.S.).

\subsection{Analyse des ZDF-Berichts zur Grass-Debatte}

Der als zweiter Stimulus verwendete Medienclip ist ein Beitrag des ZDF heute-journals, in dem sechs Personen des öffentlichen Lebens noch am Tag der Veröffentlichung des Gedichts gebeten wurden dieses zu kommentieren (vgl. Leifert 2012). Im Beitrag kommen mit Hermann Gröhe von der CDU und Andrea Nahles von der SPD ein Repräsentant und eine Repräsentantin der beiden großen Parteien, die für die U-Bootlieferung politisch verantwortlich sind, zu Wort. Präsentiert werden außerdem die Meinungen eines israelischen Historikers als Sachexperten (Moshe Zimmermann), einer israelidenmords nicht zu »Fußnoten der Geschichte« werden zu lassen (vgl. Schirrmacher 2012). 
schen Schriftstellerin (Zeruya Shaley), einem politischen Vertreter deutscher Jüd*innen (Dieter Graumann) sowie Wolfgang Gehrckes von der Linkspartei als Verteidiger von Grass. Der Medienclip wird hierbei - wie in der journalistischen Beitragsform >Bericht< üblich - durch einen Kommentar narrativ strukturiert.

Auf visueller Ebene besteht der Beitrag aus verschieden Bildmontagen des schreibenden und Tonskulpturen schaffenden Autors, des gedruckten Gedichts, eingeblendeten Zitaten und den interviewten Personen. An entsprechenden Stellen werden die sprachlichen Beiträge zudem durch Bilder von U-Booten der Dolphin-Klasse und des iranischen Präsidenten Mahmud Ahmadinedschad untermalt. Um die verbreitete Ablehnung von Grass in der politischen Öffentlichkeit zu illustrieren, werden zudem Zeitungsaufmacher eingeblendet. Hier stechen die Titelseiten der Süddeutschen Zeitung (»Ein Aufschrei«) und von Die Welt hervor, auf denen Grass als »der ewige Antisemit« bezeichnet wird.

Der Beitrag hat folgenden Wortlaut (die eingeklammerte Bezeichnung hinter den Namen sind den Angaben des Beitrags entnommen):

Sprecher: »Warum«, fragt der Dichter, »schweige ich?«, um dann in neun Strophen gekleidet, sein Schweigen zu brechen. >Was gesagt werden muss verbreitet Crass in Versform einmal um die Welt. Internationale Zeitungen drucken ein Cedicht, das wie ein Vermächtnis daherkommt. »Warum sage ich jetzt erst, gealtert und mit letzter Tinte: Die Atommacht Israel gefährdet den ohnehin brüchigen Weltfrieden?«Wirklich lyrisch ist wenig an diesem Gedicht. Mit jeder Zeile lässt sich erahnen: Hier hat sich ein politisches Statement ein Cefäß gesucht. Herausgekommen ist ein Rundumschlag gegen die Politik Israels, voll von Übertreibung und Provokation.

Moshe Zimmermann (Israelischer Historiker): Günter Grass beschrieb sein eigenes Schweigen während des Zweiten Weltkrieges. Er versucht jetzt, das zu relativieren. Er will nicht mehr verschweigen. Damals hat man verschwiegen, oder hat man ignoriert, dass man Juden ermordet hat. Jetzt will er nicht verschweigen, dass die, eben die Juden, äh versuchen, die sarmen Iraner anzugreifen, und zwar mit atomaren Bomben. Also, das ist äh seine Art, äh Wiedergutmachung für sich selbst zu leisten.

Sprecher: Er schweige nicht mehr, sagt Grass, um das Land beim Namen zu nennen, in dem eine geheime nukleare Bedrohung heranwächst: Israel. »Und, zugegeben, ich schweige nicht mehr, weil ich der Heuchelei des Westens überdrüssig bin.« 
Dieter Graumann (Zentralrat der Juden in Deutschland): Ich finde, das ist ein Pamphlet von Aggressivität und von Agitation. Ich [sic!], es ist ein Vermächtnis von Verirrung und von Verwirrung. Cünter Grass verharmlost das beinharte, brutale Terrorregime in Teheran und auf der anderen Seite denunziert und dämonisiert er Israel.

Sprecher: Grass - darin sind sich fast alle einig - verdreht Bedrohung und Bedrohte. Den iranischen Präsidenten nennt er einen Maulhelden, dessen Volk ausgelöscht zu werden droht, vom atomaren Erstschlag Israels. Und Deutschland mache sich mitschuldig, wenn es U-Boote an die Atommacht Israel liefere.

Hermann Gröhe (CDU-Generalsekretär): Es verkennt völlig die Situation, dass ein nach Atomwaffen greifender Iran, das Existenzrecht Israels bestreitet, den Holocaust leugnet und sich internationaler Kontrolle seiner Kernenergie-Konzepte verweigert.

Andrea Nahles (SPD-Generalsekräterin): Ich denke, es gibt 'ne reale Bedrohung Israels durch den Iran und von daher, äh glaube ich, dass er sich hier wirklich äh schlicht vergaloppiert hat.

Sprecher: »Das Schweigen sei zur Lüge geworden«, textet Grass. Israels nukleares Potential müsse endlich kontrolliert werden.

Zeruya Shaley (Israelische Schriftstellerin [auf hebräisch mit Untertiteln]): Ich war extrem traurig und frustriert, als ich das Gedicht las. Ich habe das Gefühl, das Herr Grass nicht vertraut ist mit der Situation in unserer Region. Da ist ein großes Missverständnis in diesem Gedicht.

Sprecher: Mit dem, was er mit letzter Tinte verfasst, droht sich ein Nobelpreisträger um seinen Ruf zu schreiben. Unterstützung für Crass muss man lange suchen.

Wolfgang Gehrcke (Fraktion »Die Linke«): Man muss fair und sauber mit Israel umgehen, man muss fair und sauber mit dem Problem äh umgehen, und man muss immer sich die Frage stellen: Was hilft eigentlich meinen Freunden in Israel? Ich geb ihnen das wichtigste, was ich hab: Meine kritische Solidarität. Und das tut Grass auch.

Sprecher: Er schweige nicht mehr, sagt Günter Crass. Als Drohung war das aber eigentlich nicht gemeint. 


\subsubsection{Konstative, evaluative und expressive Äußerungen}

Wie auch bei der inhaltlichen Analyse des Gedichts, so sollen im Folgenden die Sprechakte der verschiedenen zu Wort kommenden Personen analysiert gehen. Von Interesse ist hierbei insbesondere, auf welche Weise sich Widerspruch gegen das Gedicht bzw. Grass artikuliert und auf welche Weise es verteidigt wird.

Der Kommentator stellt das Gedicht kurz vor, paraphrasiert, zitiert und kommentiert es stellenweise, und fasst die Reaktionen auf das Gedicht zusammen. Bezüglich des Gedichts wird festgestellt, dass es als Form der politischen Meinungsäußerung verstanden werden sollte und bezüglich dessen öffentlicher Resonanz, dass wenige Personen Grass unterstützen. Gemessen an den öffentlichen Reaktionen wird das Gedicht als rufschädigend beurteilt. Hinsichtlich der Bewertung des Gedichts bleibt der Kommentator größtenteils uneindeutig. Die Beschreibung des Texts als »Rundumschlag«, »Übertreibung « und »Provokation « können sowohl als negative Bewertung oder als Würdigung verstanden werden: ein Rundumschlag kann sich ziellos gegen alles und jede*n wenden, ist dadurch mindestens ungenau und unfair. Als ein nach allen Seiten in einem Kreis geführter Schlag steht ein Rundumschlag jedoch zugleich für Mut und für virtuose Kampfkunst. Die gleichen Ambivalenzen gelten für die Wörter »Übertreibung« und »Provokationen«. Übertreibungen können unsachgemäß sein, manchmal aber auch Dinge auf den Punkt bringen. Provokationen können Mittel der Kritik von mutigen Tabubrecher"innen sein, wie auch ein Ausdruck von Aggressivität. Wenn auch in diesen Hinsichten zweideutig, so ist allerdings unzweifelhaft, dass der Kommentator in seinen letzten Worten Grass gegen (Antisemitismus-)Vorwürfe explizit in Schutz nimmt. Denn das Gedicht sei »eigentlich«nicht »als Drohung [...] gemeint «. Damit wird der mit dem Gedicht erhobene Anspruch wahrhaftig $\mathrm{zu}$ sprechen unterstrichen, und Grass zugleich schlechte Intentionen abgesprochen. Hiermit korrespondiert, dass der Kommentator den Gegenstand des Gedichts als die "Politik Israels« bestimmt und von einer im "geheimen wachsenden nuklearen Bedrohung « nicht im Konjunktiv, sondern im Modus des Indikativs spricht, wodurch eine rationalisierende Vereindeutigung des Gedichts vorgenommen wird.

Die Äußerungen des israelischen Historikers Moishe Zuckermann zeichnen sich vor allem durch ein ironisches Spiel mit Worten aus. Das Vokabular vergangenheitspolitischer Debatten wird hierbei aufgenommen und auf 
entfremdende Weise verwendet. So ist es zum Beispiel unüblich von einer »Wiedergutmachung für sich selbst « $z u$ sprechen, da diese üblicherweise für andere geleistet wird. Das Wort »relativieren« wird üblicherweise als »Herabspielen« verstanden, aber von Zuckermann im Sinne eines >dem Schweigen etwas entgegensetzen verwendet. Hieraus ergibt sich eine direkte und eine indirekte Aussage. Zum einen wird die vermutete Intention des Literaten lediglich paraphrasiert: Grass habe früher zu Verbrechen geschwiegen, wolle dies aber nicht mehr tun und dadurch eine Widergutmachung leisten. ${ }^{7}$ Zum anderen wird auf indirekte Weise eine Kritik an Grass geübt, indem nahegelegt wird, dass der Autor versuche, durch sein Gedicht, das eigene Schweigen während des Nationalsozialismus in seiner Bedeutung zu schmälern, indem er heute eine Täter-Opfer-Umkehr betreibe. Dies wird deutlich, wenn Zuckermann ironisch davon spricht, dass Grass davon ausgehe, dass »eben die [vormals Verfolgten, Anm. M. H.] Juden, äh versuchen, die sarmen Iraner anzugreifen«. Der Beitrag Zuckermanns lässt sich zusammenfassend im Sinne Habermas als Versuch verstehen, einen »therapeutischen Diskurs« (Habermas 1971: 118) anzustoßen, indem ein unbewusstes Motiv ans Licht gebracht wird. Dadurch wird zugleich Grass' Neutralität infrage gestellt.

Dieter Graumann rekurriert in seinem Widerspruch insbesondere auf normative Aspekte. Der Begriff »Agitation« qualifiziert hierbei die Schrift als Form politischer Hetze. Hiermit wird das Gedicht als Verstoß gegen Normen des zivilen Diskurses moralisch diskreditiert. Der Verweis auf ein »denunzierendes«, wie auch »dämonisierendes« Verhalten von Grass problematisiert dessen Äußerungen als verleumdend. Ebenso normativ ist die Kritik einer Verharmlosung des »Terrorregime[s] in Teheran«, wodurch den politischen Handlungen der Führungsriege des Irans ihre Legitimität abgesprochen wird. Zum anderen wird Grass Kompetenz als Sprecher infrage gestellt, wenn das Gedicht als Ausdruck von »Verirrung und Verwirrung«, also eines kognitiven Defizits, beschrieben wird. Hiermit wird der Autor des Gedichts als unzurechnungsfähig dargestellt.

Hermann Gröhe, wie auch Andrea Nahles, äußern in ihren Kommentaren vor allem Formen des inhaltlichen Widerspruchs: nicht von Israel gehe eine Gefahr aus, sondern vom Iran. Gröhe betrachtet Grass' Darstellung Israels als

$7 \quad$ Hierbei wird sich unausgesprochen auf die Veröffentlichung von Grass' autobiographischem Werk »Beim Häuten der Zwiebel « (vgl. Grass 2006) bezogen, in dem der Literat seine Mitgliedschaft in der Waffen-SS erstmals öffentlich eingestanden hatte. 
Ausdruck eines Fehlurteils (Grass »verkennt völlig die Situation«). Nahles hingegen betrachtet dessen Darstellung als Resultat von bloßer Unbedachtheit, wenn sie feststellt, dieser habe "sich schlicht vergaloppiert«. Hier dokumentiert sich die in Kapitel 2.7 beschriebene Abwehrhandlung des control-denials, im Kontext dessen antisemitisches Handeln als akzidentelles Handeln dargestellt wird. Zugleich stellen beide fest, dass für Israel vom Iran eine Gefahr ausgehe.

Auch die Schriftstellerin Zeruya Shaley weist darauf hin, dass die Grass'sche Darstellung Israels falsch sei. Sie betrachtet die Darstellung als mögliches Resultat von Unwissen (Grass sei nicht »vertraut« mit der Situation, das Gedicht beruhe demnach auf einem »Missverständis«). Shaley ist die einzige Person, die ihre Position mit expressiven Elementen der Selbstoffenbarung verbindet: statt Grass Position normativ zu kritisieren, stellt sie fest, dass sie »extrem traurig und frustriert« bei der Lektüre gewesen sei.

Wolfgang Gehrcke fokussiert demgegenüber zum einen auf die Frage, ob das Gedicht moralisch angemessen sei und rechtfertigt es, wenn er behauptet, dieses gehe »fair und sauber « mit Israel um. Zum anderen unterstreicht er Grass' Wahrhaftigkeitsanspruch. Das Gedicht sei ein Ausdruck »kritische[r] Solidaritäđ«. Gehrcke bestätigt also die von Grass verwendete Selbstdarstellung als mit Israel solidarisch verbundener Kritiker. Hierbei kommt es also zu einer Rechtfertigung des Gedichts als Ganzem.

\subsubsection{Zusammenfassung}

Zusammenfassend lässt sich feststellen, dass sich im Medienclip mehrheitlich anti-antisemitischer Widerspruch, teils in polemischer, teils sachlicher Form dokumentiert und hinsichtlich der Frage, ob Grass' Gedicht als antisemitisch zu bewerten ist, kein Urteil gefällt wird. Zugleich wird durch die Visualisierung davon, dass Grass im Diskurs auch als »ewiger Antisemit« bezeichnet worden war, darauf verwiesen, dass der Autor auch öffentlich geschmäht wurde. ${ }^{8}$

8 Hiergegen könnte eingewendet werden, dass Henrik M. Broder den Begriff des »ewigen Antisemiten« in analytischer Absicht für die Beschreibung eines Prototyps gebildeter Antisemiten entwickelt hat und die Bezeichnung als ironische Wendung des Stereotyps des sewigen Juden $<$ benutzt. (vgl. Broder 2005). Allerdings erschließt sich diese Bedeutungsebene nicht aus der Darstellung des ZDF-Beitrags. 
Fünf der sechs interviewten Personen artikulieren Widerspruch durch das Hinterfragen konstativer Behauptungen von Grass, indem betont wird, dass Israel nicht den Iran bedrohe bzw. die Bedrohungslage verkehrt dargestellt werde. Damit wird insbesondere das von Grass gezeichnete TäterOpfer-Schema infrage gestellt. Für diese Falschdarstellung ursächlich werden Fehlurteile (Gröhe), Unwissen (Shaley), Unbedachtheit (Nahles) und kognitive Inkompetenz (Graumann) genannt. Ein Akteur legt nahe, dass Grass' Handeln von unbewussten Motiven geleitet sei (Zuckermann). Demgegenüber unterstreichen zwei Akteure (Kommentator und Gehrcke), dass Grass' Gedicht Ausdruck von wahrhaftigem, d.h. vor allem nicht-bösartigem Handeln sei. Evaluativ äußern sich insbesondere Gehrcke und Graumann. Während ersterer Grass attestiert »fair« mit Israel umzugehen, verurteilt letzterer das Gedicht.

Die ungleiche Verteilung zwischen den Kritiker*innen und einem Verteidiger von Grass kann für die Debatte als repräsentativ bezeichnet werden. Die vom Kommentator artikulierte Feststellung, dass man Unterstützer*innen »lange suchen« müsse, ist allerdings insofern irreführend, da sich selbstverständlich viele Menschen hätten finden lassen, die Grass Äußerungen positiv bewerteten. Insofern kann die redaktionelle Auswahl der Sprecher*innen des Beitrags nicht als neutral, sondern als selektiv bezeichnet werden. Die Darstellung der öffentlichen Meinung basiert hierbei auf der Repräsentation des Meinungsspektrums der demokratischen Elite. Insgesamt lässt sich festhalten, dass sich im ZDF-Beitrag Argumentationsmuster, die für die öffentliche Debatte insgesamt prägend waren, widerspiegeln. Hierzu gehören insbesondere inhaltlicher und normativer Widerspruch und psychologische Deutungen des Grass'schen Verhaltens.

\subsection{Zwischenfazit}

In diesem Kapitel ging es darum, den zweiteiligen Grundreiz der Untersuchung inhaltlich zu analysieren, um damit eine Folie vorzubereiten, vor deren Hintergrund die Reaktionen der Teilnehmer*innen der Untersuchung interpretiert und auch nach ihrer inhaltlichen Angemessenheit bewertet werden können. Hinsichtlich des Grass'schen Gedichts wurde hierbei zunächst argumentiert, dass es sich bei »Was gesagt werden muss« zwar um ein politisches Gedicht handelt, dass es aufgrund seiner besonderen, hybriden Form aber auch mit nicht-literarischen Mitteln kritisiert werden kann. Im Hinblick auf die im Gedicht entwickelten Behauptungen wurde argumentiert, dass diese 
überwiegend als falsch oder als verzerrte Darstellung politischer Realität(en) zu bezeichnen sind. Darüber hinaus dokumentieren sich im Gedicht verschiedene antisemitische Strukturmuster und Stereotype:

- eine dichotome Konstruktion von Täter- und Opfergruppen

- eine personalisierende Erklärung bestehender Gefahren im Atomstreit zwischen Israel und dem Iran

- das antisemitische Stereotyp von Israelis, als Gefahr für die Menschheit

- eine argumentative Struktur, die für aufrechnende Formen des Antisemitismus typisch ist

- eine damit einhergehende Inszenierung als Tabubrecher, die durch verschiedene Formen expressiver Äußerungen über das eigene Schweigen konturiert wird.

Der ausgesprochen starke Appellcharakter des Gedichts, der darauf hinausläuft, auch andere Deutsche zu einer >Kritik« Israels zu ermuntern, kommt hierbei insbesondere durch Bezüge auf das sprachliche Register des Holocaustdiskurses zustande. Dementsprechend verweist Grass in der fünften Strophe auf »ureigene Verbrechen, die ohne Vergleich sind (Grass 2012), deren Thematisierung allerdings nicht für sich steht, sondern vor allem als Ausgangspunkt für die Mahnung dient, dass die Deutschen sich als Mittäter eines israelischen Verbrechens völlig neuen Maßstabs schuldig machen könnten.

Demgegenüber wird im ZDF-Bericht ein Ausschnitt aus der GrassDebatte dokumentiert. Die Position der journalistischen Beitragsmacher"innen ist hierbei als ambivalent zu bezeichnen, insofern der Beitrag sprachliche Wendungen benutzt, die sowohl als Kritik, wie auch als Würdigung des Grass'schen Debattenbeitrags interpretiert werden können. Inhaltlich werden vor allem Positionen dargestellt, die das Gedicht ablehnen. Grass wird von diesen Interviewten nicht explizit Antisemitismus vorgeworfen, sie rekurrieren aber teilweise auf Argumentationen, die eine solche Kritik ausmachen könnten. Zugleich wird klar, dass Antisemitismusvorwürfe in der öffentlichen Debatte formuliert wurden.

Werner Bergmann hat in seine Analyse antisemitischer Konflikte festgestellt, dass in Antisemitismuskonflikten durch die öffentlichen Medien, den üblicherweise Vertrauen geschenkt wird, ein »einflußreicher Realitätsvorschlag" vorgenommen wird (Bergmann 1997: 37). Mit van Dijk lässt sich ergänzen, dass es die demokratischen Eliten sind, die in vielerlei Hinsicht 
Ansichten "vorformulieren«, die dann zu einer "popular currency« werden (vgl. van Dijk 1992: 88). Dementsprechend wäre es naheliegend anzunehmen, dass die Teilnehmer"innen der vorliegenden Untersuchung positiv an die Kritik des Gedichts anschließen. Dies ist aber, wie im folgenden Kapitel deutlich werden wird, meist nicht der Fall. 